\title{
CAMBIAMENTI CLIMATICI E CONSEGUENZE SUL DRENAGGIO E L'IRRIGAZIONE
}

\author{
Giuseppe Taglioli, Riccardo Alessandri
}

\section{INTRODUZIONE}

\section{Introduction}

\subsection{Il CLIMA DELLA DISTRIBUZIONE}

\subsection{Climate}

Ormai da diversi anni viene messa in evidenza la tendenza verso modificazioni climatiche tali da incidere sul problema idrico in termini sia di carenza che di eccesso, e quindi con il coinvolgimento diretto del settore agricolo, in particolare per quanto concerne il drenaggio e l'irrigazione.

Il legame fra clima e agricoltura è di fondamentale importanza non solo per gli effetti immediati sulle produzioni ottenibili ma anche per l'adeguamento degli impianti ai mutati parametri climatici, nell'ambito di una corretta pianificazione dell' agricoltura del futuro.

Diversi studi [8] hanno messo in evidenza come, dalla fine del XIX secolo, a livello mondiale si siano registrati significativi cambiamenti climatici. In particolare sono aumentate le temperature della superficie del mare di $0,4-0,8^{\circ} \mathrm{C}$, dell' aria sulla superficie del mare di $0,4-0,7^{\circ} \mathrm{C}$, dell' aria sulla superficie terrestre di $0,4-0,8^{\circ} \mathrm{C}$; si è verificato un notevole ritiro dei ghiacciai alpini; la copertura nevosa primaverile nell'emisfero nord è calata del $10 \%$ dal 1987 rispetto alla media 1966-1986; si sono incrementate le precipitazioni alle alte e medie latitudini, molte delle quali caratterizzate da forte intensità $[6,9]$.

In Italia [7], rispetto al ventennio 1960-1979, la temperatura ha subito un incremento notevole, in particolare per le temperature massime sull'intero paese $\left(+0,6-0,7^{\circ} \mathrm{C}\right)$ e le minime per quel che riguarda le regioni del Nord nell' arco dell' anno $\left(+0,7-0,8^{\circ} \mathrm{C}\right)$. L'estate è la stagione che ha presentato maggior rialzo termico rispetto al ventennio precedente.

Per quanto riguarda ad esempio l'analisi dell'ultimo ventennio di precipitazioni ricavata da dati forniti dall'amministrazione provinciale di Ferrara (figg. 1-2)

Memoria presentata il 10.05.2006; accettata il 16.05.2008 emerge che, rispetto al ventennio precedente, piove di meno su tutto il paese e in ogni periodo dell' anno, con una diminuzione nazionale media del $14 \%$ verosimilmente dovuta, oltre ai noti fattori antropici, ad una minor frequenza di passaggio di perturbazioni sul nostro paese [8], ma con un incremento della violenza dei rovesci.

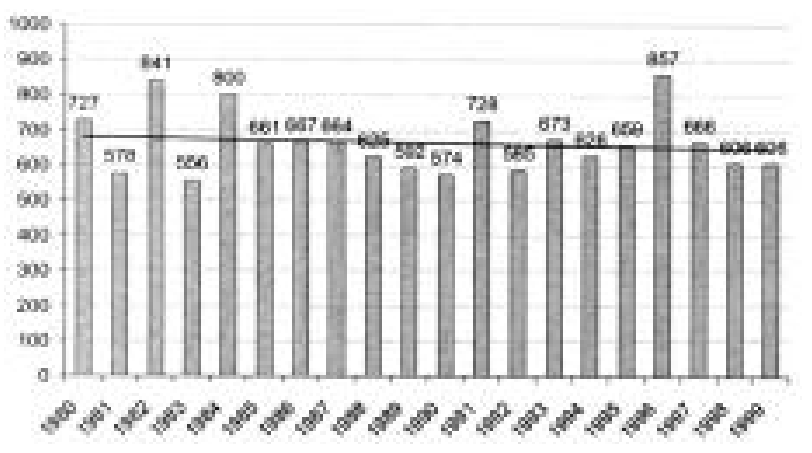

Fig. 1 - Precipitazioni annuali in Italia.

Fig. 1 - Annual precipitation in Italy.

Per quanto riguarda l'analisi climatica McKee et al. (1993), utilizzando l'indice SPI (Standardized Precipitation Index), hanno calcolato il deficit di precipitazione per diverse scale dei tempi: 1, 3, 6, 12, 24 e 48 mesi [11]. Ognuna di queste scale riflette l'impatto della siccità sulla disponibilità di differenti risorse d'acqua. L'umidità del suolo risponde alle anomalie di precipitazione su scale temporali brevi, mentre l'acqua nel sottosuolo, fiumi e invasi, tendono a rispondere su scale oggettivamente più lunghe.

L'indice necessita per il suo calcolo dei soli dati di precipitazione. Esso è calcolato considerando la deviazione della precipitazione rispetto al suo valore medio su una data scala temporale, divisa per la sua deviazione standard. Dato che la precipitazione non è normalmente distribuita, almeno su scale temporali minori dell'anno viene eseguito un aggiustamento della variabile in modo che lo SPI abbia distribuzione gaussiana con media nulla e varianza unitaria così da confrontare i valori dell'indice calcolati per diverse 


\begin{tabular}{|l|l|}
\hline Valore SPI & Classe \\
\hline$>2$ & Piovosità estrema \\
\hline Da 1.5 a 1.99 & Piovosità severa \\
\hline da 1 a 1.49 & Piovosità moderata \\
\hline da 0 a 0.99 & Piovosità lieve \\
\hline da 0 a -0.99 & Siccità lieve \\
\hline da -1 a -1.49 & Siccità moderata \\
\hline da -1.5 a -1.99 & Siccità severa \\
\hline$<-2$ & Siccità estrema \\
\hline
\end{tabular}

TABELla 1 - Classi di piovosità in base all'indice SPI.

TABLE 1 - Precipitation class of SPI.

regioni. Inoltre, esso consente di considerare periodi umidi e siccitosi nello stesso modo.

Nella tabella 1 sono mostrate le categorie di siccità e piovosità in funzione dei valori dell'indice.

Un valore inferiore a zero dell'indice quantifica l'entità del deficit idrico nel periodo considerato, mentre la sua permanenza su valori consecutivamente negativi permette di valutare la durata dell'evento siccitoso.

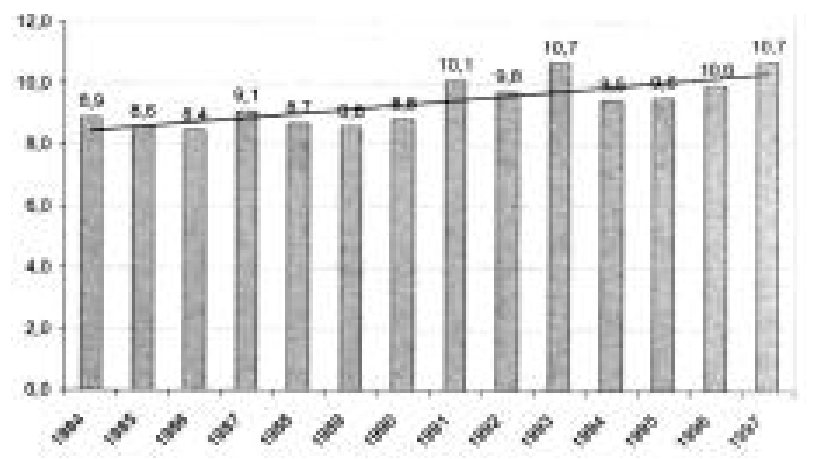

Fig. 2 - Intensità di pioggia annuali in Italia $[\mathrm{mm} /$ giorno piovoso].

Fig. 2 - Annual precipitation intensity [mm/raining day].

\subsection{ALCUNI ASPETTI TECNICI CONNESSI}

\subsection{Some related techniques aspect}

Per la progettazione delle reti drenanti sia di tipo superficiale che sotterraneo, in particolare per stabilire l'interdistanza dei dreni, occorre valutare la portata che deve essere smaltita dall'impianto, sotto forma di spessore idrico giornaliero ( $\mathrm{m} /$ giorno).

La distanza tra i dreni può essere infatti calcolata (per un terreno formato da due strati di diversa permeabilità e in moto permanente), attraverso l'espressione dell'olandese Hooghoudt (utilizzabile sia per il drenaggio superficiale che sotterraneo):

$$
L^{2}=\frac{(8 \cdot \mathrm{Ki}-\mathrm{Se} \cdot H)+\left(4-\mathrm{Ks}-H^{2}\right)}{q}
$$

dove: $L$ è la distanza tra i dreni (in $\mathrm{m}$.), $K$ è il coefficiente di filtrazione relativo allo strato che si considera (m/giorno), $H$ è il carico idraulico, in corrispondenza della mezzeria dei dreni $(\mathrm{m}), q$ è la portata da smaltire (m/giorno), Se è lo spessore "equivalente"(m) $[12,13]$.

Senza entrare nel merito dei diversi approcci utilizzati nelle progettazioni delle reti drenanti non v'è dubbio che l'analisi della piovosità su basi statistiche estesa ad un periodo di osservazioni molto lungo è di estrema utilità per poter calcolare le intensità di pioggia (rapporto fra l'altezza di precipitazione e il tempo in cui queste si sono verificate) necessarie per determinare la portata da smaltire con le reti drenanti. La sua espressione è: $\mathrm{h}=\mathrm{at}^{\mathrm{n}}$, nota anche come "curva di possibilità pluviometrica", dove h rappresenta l'altezza di precipitazione, t è la sua durata, a ed n sono due parametri che caratterizzano la curva e dipendono dalla climatologia locale, con $\mathrm{n}$ sempre inferiore a 1 e superiore a 0 .

Sulla base di questa espressione è possibile calcolare l'intensità della precipitazione tenendo conto delle piogge di durata di 1, 2, 3, 4, 5 giorni che possono verificarsi con maggiore frequenza ed é possibile costruire una serie di casi critici. Non si scelgono normalmente $i$ primi casi critici, per non dover dimensionare le reti drenanti in proporzione ad eventi che hanno una scarsa probabilità che si verifichino, bensì il quarto, quinto caso critico: le colture non subiscono infatti danni produttivi se non, mediamente, dopo circa tre giorni in condizioni di asfissia radicale, quindi a base dei calcoli si può assumere una pioggia giornaliera, e quindi la corrispondente intensità, dividendo per tre quella di tre giorni.

Attualmente, nei climi mediterranei, si fa normalmente riferimento ad una pioggia di 3 giorni di 60$70 \mathrm{~mm}$, pari a circa $20 \mathrm{~mm} /$ giorno; valutando poi che questa pioggia giornaliera non sarà per la sua totalità soggetta all'azione dei dreni (a causa del ruscellamento superficiale, evaporazione, trattenuta da parte della vegetazione) si usa considerare, nel calcolo, una portata inferiore di oltre il $50 \%$, e quindi di circa 8$10 \mathrm{~mm} /$ giorno $[12,13]$.

Per il distanziamento dei dreni attualmente vengono fornite le seguenti indicazioni:

$\begin{array}{lll}\text { Tipo di terreno } & \text { Drenaggio sotterraneo } & \text { Drenaggio superficiale } \\ \text { argilloso } & 6-12 \mathrm{~m} & 20-25 \mathrm{~m} \\ \text { medio impasto } & 12-18 \mathrm{~m} & 25-40 \mathrm{~m} \\ \text { sabbioso } & 18-24 \mathrm{~m} & 40-60 \mathrm{~m}\end{array}$

In questo lavoro, in un comprensorio di bonifica ferrarese (fig. 3), vengono esaminati e confrontati i dati climatici in un intervallo temporale sufficientemente lungo, allo scopo di ricavare indicazioni tecniche relative all'adeguatezza degli attuali impianti aziendali di drenaggio ed anche alle mutate esigenze irrigue delle colture.

\section{MATERIALI E METODI}

\section{Materials and methods}

Le fasi in cui si articola il presente lavoro sono così strutturate: 


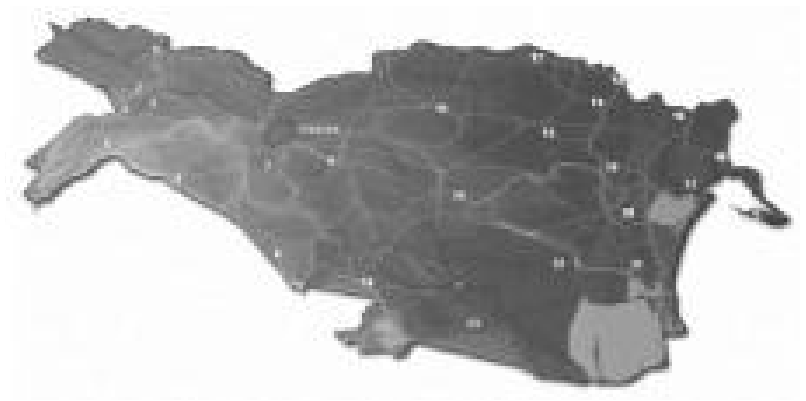

Fig. 3 - Stazioni pluviometriche nella provincia di Ferrara.

Fig. 3 - Meteorological stations of Ferrara.

- analisi dei dati meteorologici degli ultimi sessantacinque anni (1939-2003) relativi al comprensorio del Consorzio Valli di Vecchio Reno (che gestisce i dati), rappresentato dalle stazioni pluviometriche di Torniano-Spinazzino, altitudine $10 \mathrm{~m}$ s.l.m., (stazioni 6 e 8 in fig. 3 );

- digitalizzazione e successiva analisi delle strisciate pluviometriche relative a tale comprensorio;

- costruzione di indici climatici (SPI) e delle curve di possibilità climatica $\left(\mathrm{h}=\mathrm{at}^{\mathrm{n}}\right)$.

Il Consorzio Valli di Vecchio Reno, gestisce un comprensorio di 43.230 ha ricadenti in parte nella Provincia di Ferrara (Comuni di Argenta, Bondeno, Cento, Ferrara, Mirabello, Poggio Renatico, S. Agostino e Vigarano Mainarda), in parte nella Provincia di Bologna (Comuni di Baricella, Molinella e S. Giovani in Persiceto) e in parte in provincia di Modena (Comune di Finale Emilia) [1, 2, 3, 14].

Per l'analisi climatica, dei vari "indici di siccità" disponibili, si è utilizzato lo SPI.

\section{RISULTATI E DISCUSSIONE}

\section{Results and discussion}

\subsection{ANALISI CLIMATICA DEL COMPRENSORIO CONSIDERATO: LA PIOVOSITÀ}

\subsection{Climatic analysis of the studied district:} The Rainfall

È interessante osservare che il minimo e il massimo annuale di pioggia $(288,2 \mathrm{~mm}$ e $965,8 \mathrm{~mm})$ appartengono entrambi agli ultimi $10 \mathrm{anni}$, rispettivamente al 1993 e al 1996 (fig. 4). Il 2002 e il 2003 (tanto discussi per la eccessiva piovosità dell'uno e la siccità dell'altro) hanno fatto registrare il $30 \%$ in più rispetto alla media per il 2002 e poco più del $10 \%$ in meno per il $2003[4,5]$.

Raggruppando i dati per stagione si nota come, a parte l'estate che ha una linea di tendenza praticamente orizzontale, le altre stagioni mostrino una tendenza verso una riduzione della piovosità.

Il grafico relativo al periodo di un mese (fig. 5) riporta la situazione della "siccità meteorologica", che

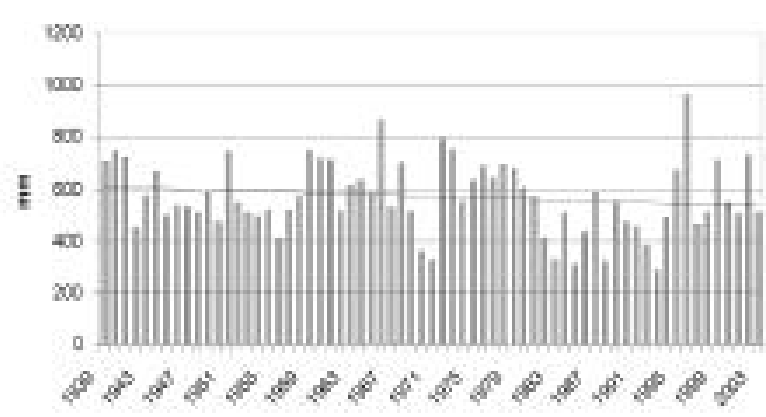

Fig. 4 - Totali annuali di pioggia dal 1939 al 2003.

Fig. 4 - Annual precipitation from 1939 to 2003.

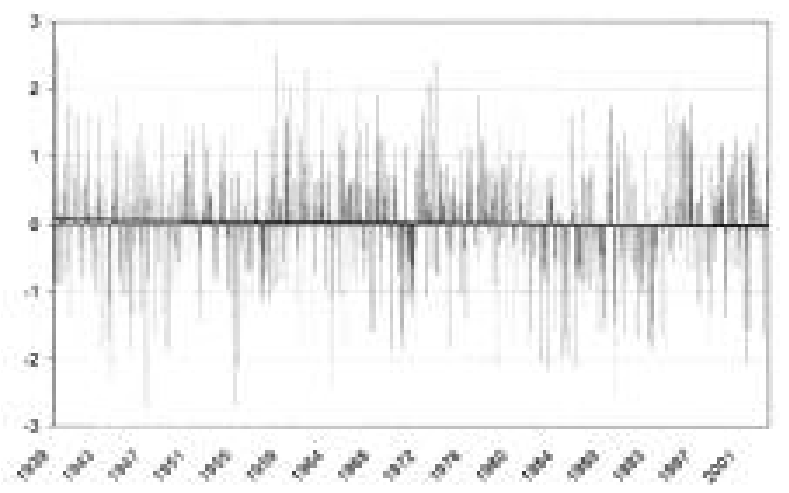

Fig. 5 - Valori di SPI calcolati su una scala temporale di 1 mese.

Fig. 5 - Values of SPI calculated with a time scale of 1 month.

mostra l'andamento dell'influenza della piovosità sull'umidità del suolo. Si noti che la linea di tendenza è leggermente inclinata, ma in maniera quasi irrilevante.

Con il periodo di riferimento di 12-24 mesi (figg. 6-7) si rientra nell'ambito della cosiddetta siccità agronomica.

L'andamento relativo al periodo di 48 mesi (fig. 8) descrive l'evoluzione della siccità idrologica, rappresentatrice delle anomalie di falda.

Si può osservare dai grafici come tutte le tendenze siano in negativo, si tende cioè a periodi mediamente

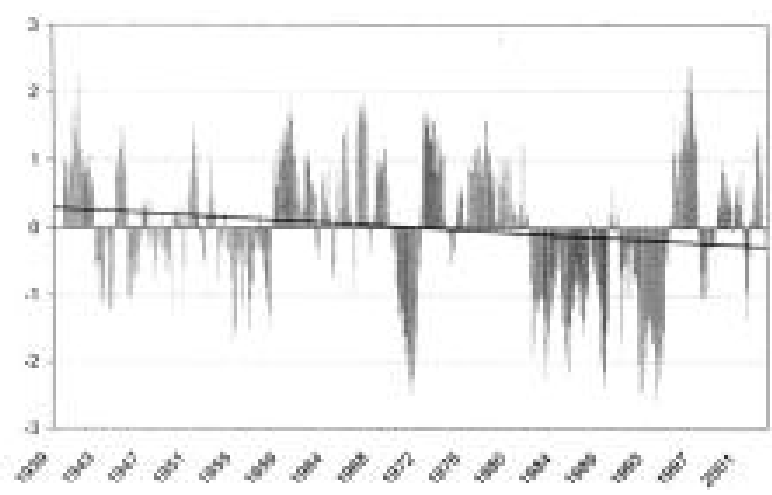

Fig. 6 - Valori di SPI calcolati su una scala temporale di 12 mesi.

Fig. 6 - Values of SPI calculated with a time scale of 12 months. 


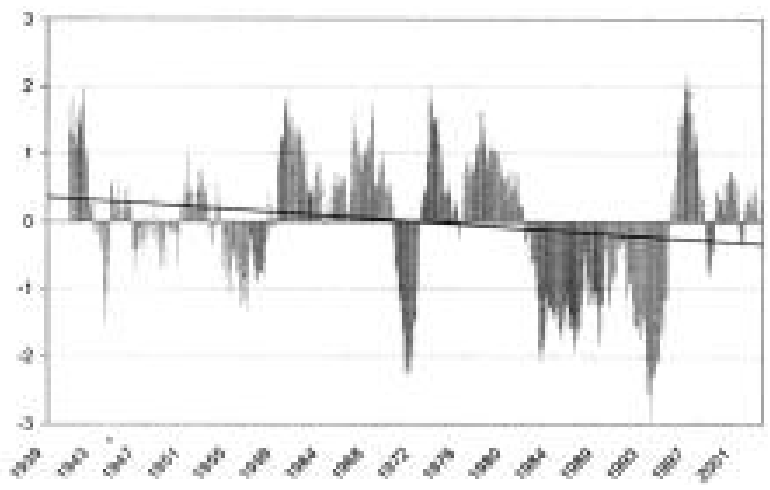

Fig. 7 - Valori di SPI calcolati su una scala temporale di 24 mesi.

Fig. 7 - Values of SPI calculated with a time scale of 24 months.

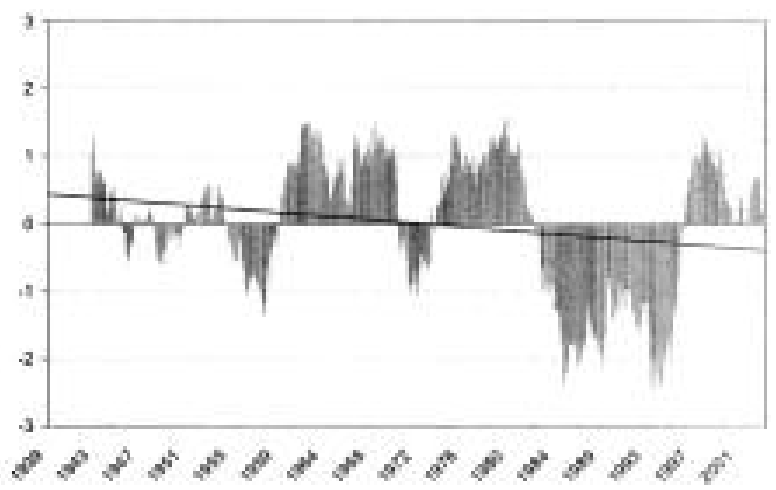

Fig. 8 - Valori di SPI calcolati su una scala temporale di 48 mesi.

Fig. 8 - Values of SPI calculated with a time scale of 48 months.

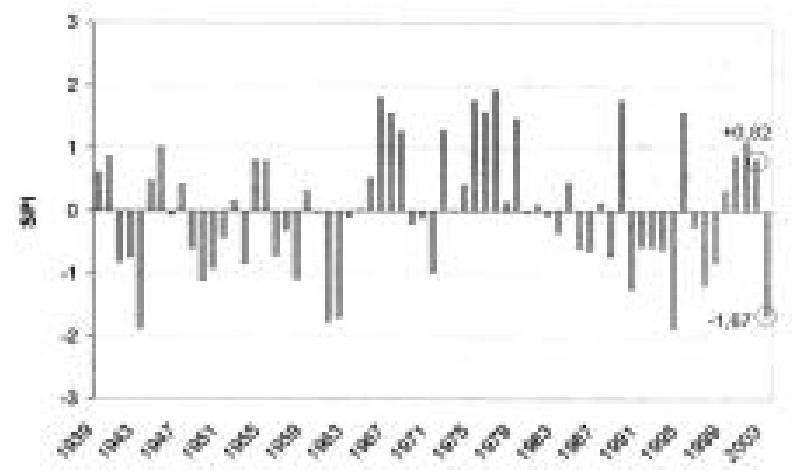

Fig. 9 - Valori di SPI del mese di Agosto su una scala temporale di 1 mese.

Fig. 9 - Values of SPI of August calculated with a time scale of 1 month.

siccitosi, ma quando ci si sposta verso scale temporali più lunghe il fenomeno si accentua. Sembra quindi che il fattore più penalizzato sia quello legato alle risorse idriche a lungo termine, ma a questo punto è opportuno focalizzare l'attenzione sui periodi stagionali. Lo SPI viene calcolato mensilmente, indipendentemente dalla scala temporale utilizzata: selezionando solo un mese per ogni anno si avrà la possibilità di vi- sualizzare meglio l'attenzione sui particolari. Un esempio è l'andamento che si ottiene per Agosto: in questo caso il valore dell'indice del mese di Agosto di ogni anno viene confrontato con quello relativo a tutti gli Agosto precedenti.

Come evidenziato nel grafico (fig. 9), si nota chiaramente il passaggio brusco 2002-2003 [4, 5], in cui si passa da un valore dello SPI di 0,82 di Agosto 2002 ad un valore di -1,67 di Agosto 2003, che corrisponde ad una situazione di "siccità severa".

La situazione descritta in quel contesto combacia con la tendenza di tutte le tipologie di siccità, ma per quanto riguarda la siccità agronomica le tendenze dell'autunno e dell'inverno evidenziano forme più lievi rispetto alla media annuale.

La situazione della siccità idrologica è quella a cui corrispondono le tendenze più marcate; meno chiara risulta invece la situazione corrispondente alla siccità meteorologica. Essa è infatti indicativa degli eventi momentanei, a breve termine e quindi anche con la variabilità più elevata. Inoltre bisogna notare che le aridità estive del nostro clima sono caratterizzate da una tendenza all'aumento di eventi a carattere temporalesco di intensità dell'ordine di poche ore, e quindi non calcolabili da questo indice che si basa su scala mensile, interconnessi da lunghi periodi siccitosi.

Si può notare come tutte le linee di tendenza, tranne quella corrispondente ad un mese, mostrino via via una maggiore pendenza con l'allungamento della scala temporale.

Secondo questa ipotesi sarebbero quindi i valori dei livelli di falda e di tutte le risorse disponibili nel lungo periodo a subire le maggiori variazioni.

La scala temporale di un mese risulta negativa: questo non è necessariamente in contraddizione col quadro globale.

Come già spiegato, infatti, i problemi estivi riguardano proprio l'aumento marcato di eventi piovosi estremi, ma con intensità talmente elevate (tempi inferiori alle 24 ore) da non poter essere calcolati da un indice di tipo mensile [11].

\subsection{GLI ALTRI FATTORI DEL CLIMA: LA TEMPERATURA}

\subsection{The other elements of climate: the temperature}

In riferimento all'intero territorio della provincia di Ferrara per il periodo 1975-2003 il dato più significativo è quello delle temperature massime, in aumento di circa $2,5^{\circ} \mathrm{C}$ come valore medio di tendenza. Le minime e le medie hanno un andamento praticamente piatto o leggermente in aumento.

La variazione termica influisce sull'entità della evapotraspirazione [10].

I valori annuali ed estivi, rappresentati nelle fig 10 11, sono stati calcolati secondo il metodo di Hargreaves (1982), che utilizza i soli dati giornalieri di temperatura minima e massima, considerando come correttivo la radiazione solare extra-terrestre in funzione della latitudine (fig. 12). 


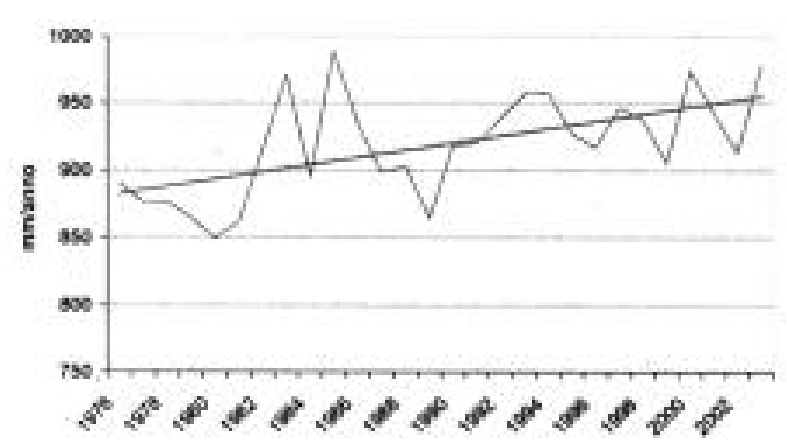

Fig. 10 - Evapotraspirazione annuale.

Fig. 10 - Annual evapotraspiration.

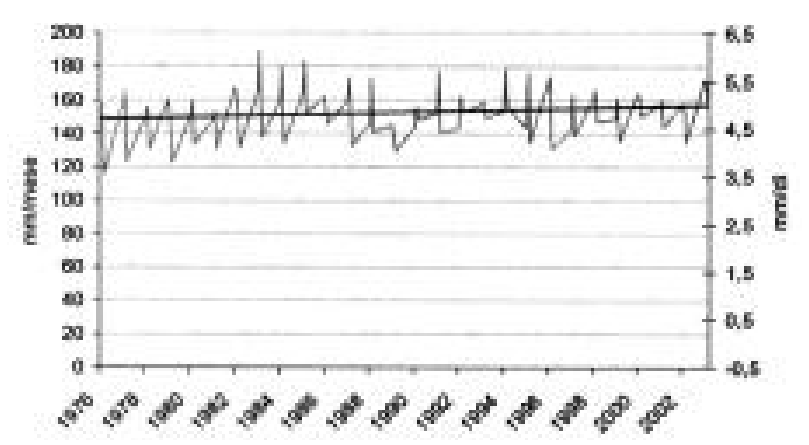

Fig. 11 - Evapotraspirazione estiva.

Fig. 11 - Summer evapotraspiration.

$$
\text { ETO }=C^{\star} R a^{*}(T+17.8)^{*} \operatorname{sqrt}(T x-T n)
$$

\section{dove:}

\section{Eto $=$ flusso evapotraspirativo in $\mathrm{mm}^{-1}$}

\section{$\mathrm{C}=$ costante empirica pari a 0.0023}

$\mathrm{Ra}=$ radiazione solare extraterrestre [mm di $\mathrm{H}_{2} \mathrm{O}$ evaporata] $\mathrm{T}=$ Temperatura media giomaliera $=(T \mathrm{x}+\mathrm{Tn}) / 2$

Fig. 12 - Formula di Hargreaves.

Fig. 12 - Expression of Hargreaves.

La fig. 13 analizza la correlazione fra pioggia-temperatura ed evapotraspirazione.

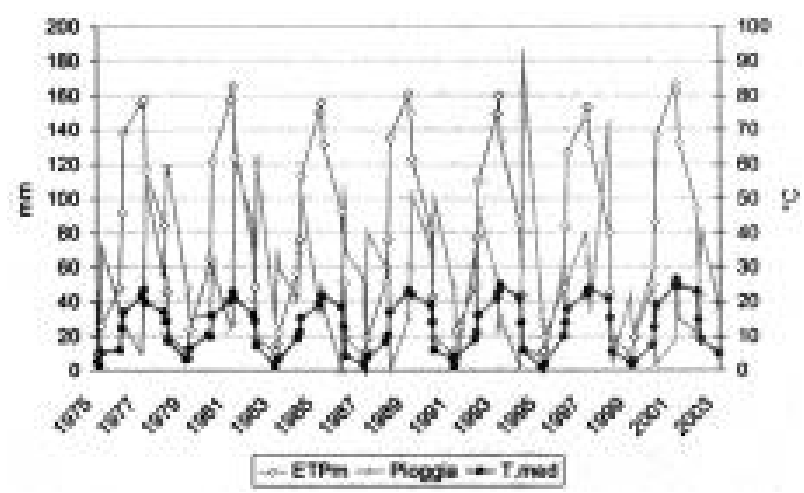

Fig. 13 - Correlazione fra Temperatura - Pioggia - Evapotraspirazione.

Fig. 13 - Connection between Temperature, precipitation and evapotraspiration.

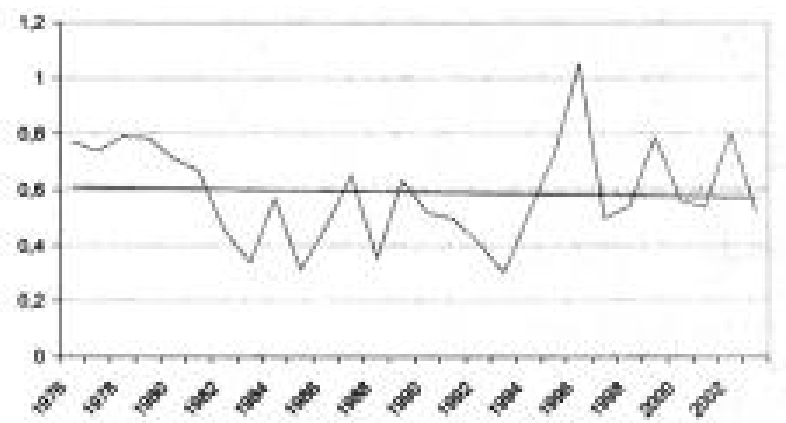

Fig. 14 - Indice di Aridità.

Fig. 14 - Drought index.

In rapporto all'irrigazione la tendenza all'aumento dell' evapotraspirazione nel periodo estivo, valutabile in circa $30 \mathrm{~mm}$, porterebbe, unitamente ad un aumento delle intensità orarie di pioggia e quindi ad una riduzione della componente "agronomicamente utile" delle piogge stesse, alla necessità di erogare volumi d'adacquamento stagionali di maggiori entità.

L'entità di tali maggiori apporti idrici (300-400 $\mathrm{m}^{3} / \mathrm{ha}$ ), nell' ambiente padano considerato, corrisponderebbero a circa il $25 \%$ dell'attuale fabbisogno irriguo medio delle colture $[12,13]$. Un indice di aridità molto semplice si ottiene dal rapporto fra i totali annuali di pioggia e quelli di evapotraspirazione i cui valori sono riportati nella figura 14 .

I valori inferiori ad 1 (la quasi totalità) indicano che il valore di ETP supera quello di precipitazione: minore è il rapporto, più alto è il divario fra l'ETP e i totali di pioggia, la soglia di aridità viene individuata per valori inferiori a 0,6: l'andamento è comunque sempre in negativo a conferma di quanto detto fino ad ora.

\subsection{IL CLIMA E IL DRENAGGIO}

\subsection{Climate and drainage}

I dati del pluviometro di Torniano sono stati utilizzati per l'indagine climatica in generale mentre, per l'elaborazione dei dati pluviometrici ai fini del calcolo dei casi critici, si è preferito fare riferimento alla banca dati del pluviografo di Benvignante (collocato sempre all'interno dello stesso comprensorio), di cui è stato possibile calcolare (a differenza di Torniano) i coefficienti $a$ ed $n$ della curva di possibilità climatica per un intero periodo di 50 anni (dal 1951 al 2003), periodo diviso, per un confronto, in due sottoperiodi di 25 anni ciascuno: 1951 al 1976 e dal 1978 al 2003.

Nella tabella 2 vengono indicati i coefficienti $a$ e $n$ per i vari tempi di ritorno, calcolati mediante la funzione di probabilità di Gumbel, nell'intero periodo di 50 anni (i valori sono stati precedentemente ragguagliati a febbraio mediante i coefficienti del Pasini).

Le curve di possibilità climatiche relative all'intero periodo e ai due distinti periodi storici sono rappresentate nelle tabelle 3-4-5 e figg. 15-16-17.

Si può notare l'aumento del coefficiente $a$ dal primo al secondo periodo, elemento che porta ad una modifi- 


\begin{tabular}{|c|c|c|c|}
\hline $\begin{array}{c}\text { Tempo di } \\
\text { Ritorno(anni) }\end{array}$ & $\begin{array}{c}\text { Caso critico } \\
\text { corrispondente } \\
\text { (approssimato) }\end{array}$ & $\mathrm{a}[\mathrm{mm} / \mathrm{h}]$ & $\mathrm{n}$ \\
\hline 5 & 11 & 10.6 & 0.448 \\
\hline 10 & 6 & 12.3 & 0.450 \\
\hline 20 & 3.5 & 13.9 & 0.450 \\
\hline 35 & 2.5 & 15.8 & 0.453 \\
\hline 50 & 2 & 16.1 & 0.456 \\
\hline 70 & 1.7 & 16.8 & 0.457 \\
\hline 100 & 1.5 & 17.7 & 0.457 \\
\hline
\end{tabular}

TABELla 2 - Coefficienti di Gumbel nel periodo totale di 50 anni (1951-2003).

TABLE 2 - Gumbel coefficients in a period of 50 years (1951-2003).

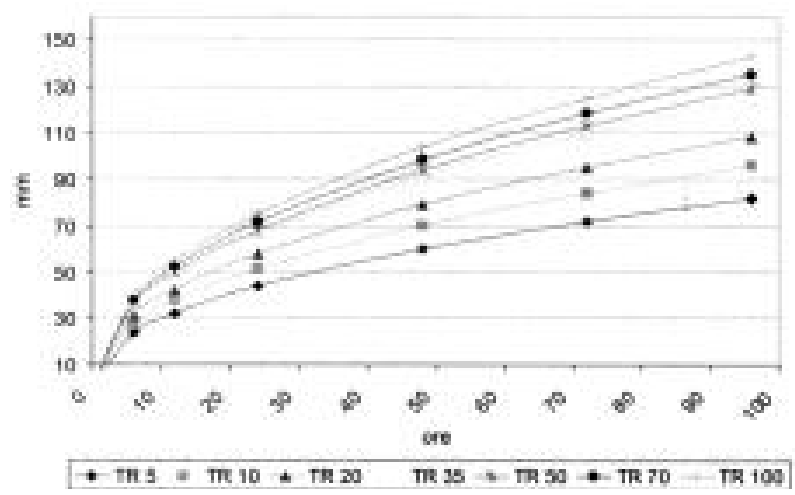

Fig. 15 - Curve di possibilità climatica del periodo di 50 anni (1951-2003)

Fig. 15 - Climatic possibility curve of the period of 50 years (19512003).

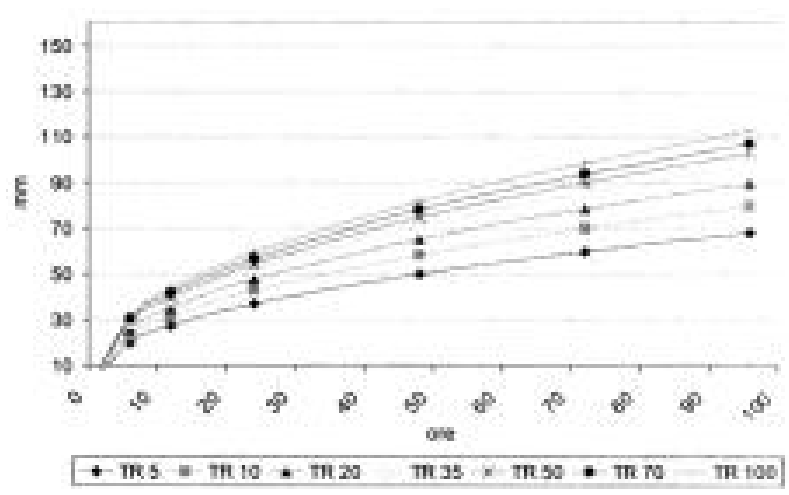

Fig. 16 - Curve di possibilità climatica del $1^{\circ}$ periodo (19511976).

Fig. 16 - Climatic possibility curve of the $1^{\circ}$ period (1951-1976).

cazione delle curve di possibilità nei due periodi storici.

Si nota chiaramente l'aumento nel secondo periodo storico dei valori di $h$, corrispondenti ad ogni tempo di pioggia in ore. Passando al calcolo del valore di $h$, elemento fondamentale per la determinazione della $q$ (portata) di progetto, come tempo $t$ è stato utilizzato quello pari a 72 ore (3 giorni).

I valori di $h$, calcolati prima per l'intero periodo, poi per i due periodi separati sono quelli evidenziati dalla tabella 5 .

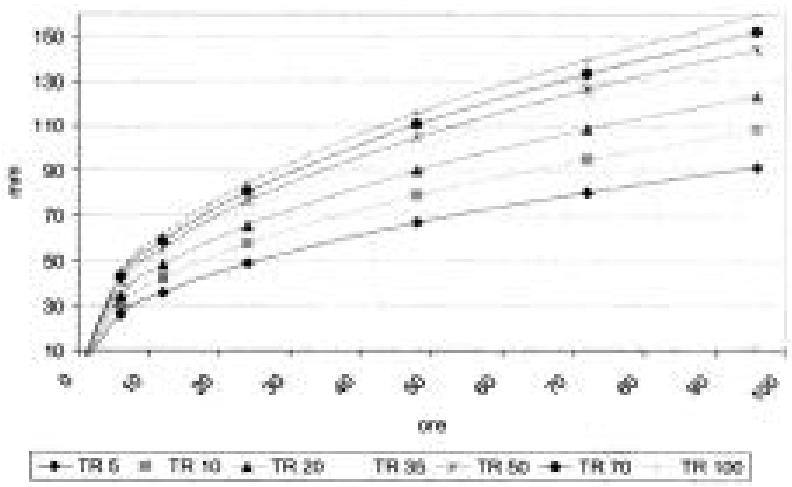

Fig. 17 - Curve di possibilità climatica del $2^{\circ}$ periodo (19782003).

Fig. 17 - Climatic possibility curve of the $2^{\circ}$ period (1978-2003).

\begin{tabular}{|c|c|c|c|}
\hline \multirow{2}{*}{$\begin{array}{c}\text { Tempo di } \\
\text { Ritorno(anni) }\end{array}$} & Caso & \multicolumn{2}{|c|}{$1^{\circ}$ periodo $(1951-1976)$} \\
\cline { 3 - 4 } & Critico & $\mathrm{a}[\mathrm{mm} / \mathrm{h}]$ & $\mathrm{n}$ \\
\hline 5 & 11.00 & 8.9 & 0.446 \\
\hline 10 & 6.00 & 10.2 & 0.450 \\
\hline 20 & 3.50 & 11.3 & 0.453 \\
\hline 35 & 2.43 & 12.2 & 0.455 \\
\hline 50 & 2.00 & 12.8 & 0.456 \\
\hline 70 & 1.71 & 13.3 & 0.457 \\
\hline 100 & 1.50 & 13.9 & 0.458 \\
\hline
\end{tabular}

TABella 3 - Coefficienti di Gumbel nel $1^{\circ}$ periodo (19511976).

TABLE 3 - Gumbel coefficients in the $1^{\circ}$ period (19511976).

\begin{tabular}{|c|c|c|c|}
\hline \multirow{2}{*}{$\begin{array}{c}\text { Tempo di } \\
\text { Ritorno(anni) }\end{array}$} & \multirow{2}{*}{$\begin{array}{c}\text { Caso } \\
\text { Critico }\end{array}$} & \multicolumn{2}{|c|}{$2^{\circ}$ periodo (1978-2003) } \\
\cline { 3 - 4 } & $\mathrm{a}[\mathrm{mm} / \mathrm{h}]$ & $\mathrm{n}$ \\
\hline 5 & 11.00 & 11.8 & 0.449 \\
\hline 10 & 6.00 & 13.7 & 0.453 \\
\hline 20 & 3.50 & 15.6 & 0.454 \\
\hline 35 & 2.43 & 17.1 & 0.456 \\
\hline 50 & 2.00 & 18.0 & 0.456 \\
\hline 70 & 1.71 & 18.9 & 0.457 \\
\hline 100 & 1.50 & 19.8 & 0.457 \\
\hline
\end{tabular}

Tabella 4 - Coefficienti di Gumbel nel $2^{\circ}$ periodo (19782003).

TABLE 4 - Gumbel coefficients in the $2^{\circ}$ period (19782003).

I valori percentuali delle variazioni di h sono dell'ordine del $40 \%$ in media fra il primo e il secondo periodo (tab. 6), corrispondenti a circa $32 \mathrm{~mm}$ di pioggia in 72 ore.

Questo risultato, in sintonia con i risultati delle analisi climatiche effettuate in precedenza, conferma la tendenza verso l'aumento delle intensità di pioggia. Passando dai valori di h ai valori di q di progetto (in $\mathrm{m} /$ dì), si ottengono i dati della tabella 7 .

Le variazioni percentuali di $q$ restano ovviamente dell' ordine del $40 \%$. 


\begin{tabular}{|c|c|c|c|}
\hline \multirow{2}{*}{$\begin{array}{c}\text { Tempi } \\
\text { di Ritorno (anni) }\end{array}$} & 50 anni & $1^{\circ}$ periodo & $2^{\circ}$ periodo \\
\cline { 2 - 4 } & $\mathrm{h}[\mathrm{mm}]$ & $\mathrm{h}[\mathrm{mm}]$ & $\mathrm{h}[\mathrm{mm}]$ \\
\hline 5 & 72.01 & 59.95 & 80.51 \\
\hline 10 & 84.28 & 69.89 & 95.08 \\
\hline 20 & 95.24 & 78.42 & 108.73 \\
\hline 35 & 109.66 & 85.40 & 120.21 \\
\hline 50 & 113.18 & 89.98 & 126.54 \\
\hline 70 & 118.61 & 93.90 & 133.43 \\
\hline 100 & 124.96 & 98.55 & 139.79 \\
\hline
\end{tabular}

TABELla 5 - Valori di h calcolati prima per l'intero periodo, poi per i due periodi separati.

TABLE 5 - $\mathrm{h}$ value, calculated for the period of 50 years and after for the two separated periods.

\begin{tabular}{|c|c|}
\hline Tempi di Ritorno (anni) & Variazioni di $\mathrm{h} \%$ \\
\hline 5 & 34.30 \\
\hline 10 & 36.05 \\
\hline 20 & 38.64 \\
\hline 35 & 40.76 \\
\hline 50 & 40.63 \\
\hline 70 & 42.11 \\
\hline 100 & 41.84 \\
\hline
\end{tabular}

TABella 6 - Variazioni percentuali di $h$.

TABLE $6-\mathrm{h}$ percentage variation.

\begin{tabular}{|c|c|c|c|}
\hline \multirow{2}{*}{$\begin{array}{l}\text { Tempi di Ritorno } \\
\text { [anni] }\end{array}$} & \multicolumn{3}{|c|}{ Portata [m/dì] } \\
\cline { 2 - 4 } & 50 anni & $1^{\circ}$ periodo & $2^{\circ}$ periodo \\
\hline 5 & 0.024 & 0.020 & 0.027 \\
\hline 10 & 0.028 & 0.023 & 0.032 \\
\hline 20 & 0.032 & 0.026 & 0.036 \\
\hline 35 & 0.037 & 0.028 & 0.040 \\
\hline 50 & 0.038 & 0.030 & 0.042 \\
\hline 70 & 0.040 & 0.031 & 0.044 \\
\hline 100 & 0.042 & 0.033 & 0.047 \\
\hline
\end{tabular}

TABELla 7 - Valori di portata relativi al periodo totale e ai 2 periodi separati.

TABLE 7 - Values of flow of the total period and of the separated periods.

Considerando però che nell'espressione dell'Hooghoudt, L è in funzione della radice quadrata di q, si può stimare che le variazioni dovrebbero portare ad una riduzione delle interdistanze tra i dreni dell'ordine del $20 \%$ in media.

Considerando le attuali interdistanze (L), sia per quanto riguarda il drenaggio sotterraneo che superficiale [13], le variazioni in metri risulterebbero quelle indicate in tabella 8 .

Le variazioni in riduzione a cui dovrebbero adattarsi i sistemi drenanti restano quindi di modesta rilevanza assoluta per terreni di tipo argilloso o franco-argilloso, mentre divengono consistenti (anche $10 \mathrm{~m}$ ) nei terreni più sciolti (sabbiosi o limoso-sabbiosi).

\begin{tabular}{|l|c|c|c|c|}
\hline Terreno & L (scoline) & var [m] & L (dreni) & var [m] \\
\hline argilloso & 20 & -4 & 10 & $-2,0$ \\
\hline franco & 30 & -6 & 14 & -2.8 \\
\hline limoso-sabb. & 40 & -8 & 16 & -3.2 \\
\hline sabbioso & 50 & -10 & 25 & $-5,0$ \\
\hline
\end{tabular}

TABELla 8 - Variazioni in $m$. delle attuali interdistanze.

TABLE 8 - Variation in $\mathrm{m}$. of the actual interdistances.

\section{CONCLUSIONI}

\section{Conclusions}

I dati storici elaborati, limitatamente all'area comprensoriale esaminata, hanno permesso di valutare la tendenza del clima e l'impatto sull'agricoltura. Lo studio ha riguardato soltanto questo territorio consortile e un numero limitato di stazioni meteo e quindi le osservazioni non possono essere generalizzate a livello territoriale più ampio, anche se sembrano in sintonia con quelle riportate in altri studi di settore.

La situazione climatica italiana mostra alcune tendenze fondamentali, in particolare un aumento delle temperature nel periodo estivo e in tutto il corso dell'anno, delle intensità di pioggia.

La situazione relativa alla zona del ferrarese considerata va vista sotto due prospettive diverse: nel breve periodo si assiste ad una sostanziale stazionarietà nei totali di pioggia e nel numero di giorni piovosi totali annuali, mentre si configura una variazione della distribuzione percentuale delle piogge di ogni stagione in rapporto al totale annuo.

Tale variazione è positiva per l'autunno e per l'inverno, negativa per la primavera e per l'estate (dal 30\% al 23\%), confermando in questo caso le tendenze nazionali. I giorni di pioggia appaiono in aumento in tutte le stagioni ad eccezione di quella estiva che ne osserva una diminuzione di oltre il $20 \%$, determinando quindi un aumento delle intensità di pioggia del $33 \%$.

In riferiemento al lungo periodo la situazione appare diversa: i totali annuali vedono una flessione del $10 \%$ suddivisa più o meno equamente in tutte le stagioni e in particolare nel periodo primaverile con percentuali di diminuzione simili a quelle nazionali $(-37,5 \%)$.

L'indice SPI ha poi confermato una tendenza verso periodi siccitosi, più marcata sul lunghissimo periodo a scapito più delle risorse idriche a lungo termine (falda) che non rispetto all'umidità del suolo in sé. Per quanto concerne le temperature la tendenza a livello nazionale mostra un aumento nelle temperature massime particolarmente evidente in estate, con una anomalia di $+0,8^{\circ} \mathrm{C}$ in positivo. Nel comprensorio considerato, la situazione mostra un aumento generalizzato per le temperature massime e le escursioni (differenza fra i massimi e i minimi), particolarmente evidente nel periodo primaverile-estivo $\left(+3^{\circ} \mathrm{C}\right.$ sulla media del periodo), mentre mostra una variazione più marcata nei mesi invernali per quanto riguarda le anomalie.

Per le evapotraspirazioni, si è osservato un aumento 
medio annuale di circa $90 \mathrm{~mm}$ e incrementi medi mensili che vanno dai $10-15 \mathrm{~mm}$ del periodo estivo-primaverile ai $2-3 \mathrm{~mm}$ di quello invernale e autunnale. Nel periodo estivo soprattutto l'aumento delle intensità di pioggia e quindi la riduzione della componente "utile" delle precipitazioni stesse in abbinamento con l'aumento delle evapotraspirazioni, corrispondenti a $30-35 \mathrm{~mm}$, porterebbe ad un necessità di incremento irriguo almeno di $300-400 \mathrm{~m}^{3} / \mathrm{Ha}$ (pari al $25 \%$ del fabbisogno irriguo medio delle colture nella zona considerata).

Lo studio delle precipitazioni nel periodo storico considerato ha poi mostrato una variazione significativa in aumento nei valori della portata di calcolo delle sistemazioni drenanti, a parità di tempi di ritorno. In particolare le altezze di pioggia in funzione del tempo, derivanti dalle espressioni relative alle curve di possibilità climatica, hanno fatto registrare variazioni in aumento intorno al $40 \%$. Tali valori, rientrando nei parametri di calcolo delle reti drenanti, hanno mostrato come l'interdistanza di tali reti, principale parametro progettuale, tenendo conto dei valori medi attualmente riscontrabili, dovrebbero essere ridotte di circa il $20 \%$. Senza queste variazioni strutturali, attualmente i sistemi di drenaggio aziendali, (indipendentemente dal fatto che siano poi attualmente concepiti in modo corretto), sarebbero ora dimensionati in relazione a un tempo di ritorno non più di 10 anni ma inferiore ai 5 anni: ciò comporterebbe quindi un raddoppio del rischio di danneggiamento alle colture. Da osservazioni generalizzate si può inoltre ritenere che le attuali reti drenanti tendono ad essere comunque sottodimensionate. L'esigenza infatti di una moderna agricoltura, che spinge ad adottare unità di coltivazione sempre più ampie e prive di ostacoli alla meccanizzazione, ha portato all'adozione di sistemazioni drenanti, in particolare quelle superficiali, che spesso non sono più sufficienti a far fronte ai nuovi eventi meteorici. Ciò non vuol dire però che, da un punto di vista economico, la riduzione delle interdistanze delle reti drenanti comporti sempre una perdita. Andrebbe infatti valutata la differenza tra i maggiori costi indotti da una riduzione di tali interdistanze (es. maggiori oneri di meccanizzazione, riduzione della superficie utile coltivabile, maggiori effetti di bordo) e i benefici relativi ai minori danni alle colture per asfissia radicale (oltre al possibile benefico effetto sulla regimazione delle acque a livello di bonifica idraulica grazie all'aumento del volume specifico di affossature). Il clima sta quindi effettivamente cambiando [8] coinvolgendo in maniera sostanziale anche le pratiche agronomiche dell'irrigazione e del drenaggio: per poter garantire nel tempo la massima produttività delle colture agrarie occorrerebbe quindi, a livello comprensoriale, verificare e poi modificare i parametri progettuali di queste due importanti operazioni agronomiche adattandole alle mutate condizioni climatiche.

\section{BIBLIOGRAFIA}

\section{References}

[1] Amministrazione Provinciale Di Ferrara, Terre ed acqua: le bonifiche ferraresi nel delta del Po, (1980).

[2] CASSA Di RisParmio Di FerRara, Storia di Ferrara: Territorio e preistoria, (2001).

[3] Cassa Di Risparmio Di Ferrara, Ferrara e la Bonifica, (1986).

[4] Rivista Dell'arpa, N. 3 Anno VI, (Maggio-Giugno 2003).

[5] Rivista Dell'arpa, N. 4 Anno VI, (Luglio-Agosto 2003).

[6] Rivista Panorama, Mondatori, (22 Aprile 2004).

[7] Giugliacci M., Abelli S., DiPIERro G., Il clima dell'Italia nell'ultimo ventennio, Collana Meteo, Alpha Tesi (Luglio 2001).

[8] Pasini A., I Cambiamenti Climatici, Bruno Mondatori, (2003).

[9] AA.Vv., Meteorologia, Atlanti Scientifici Giunti, (2002).

[10] GREPPI M., Idrologia, Hoepli, (2003).

[11] Hi-Ryong Byun (Department of Atmospheric Sciences, Pukyong National University, Republic of Korea) - DonAlD A. WILhITE (National Drought Mitigation Center, University of Nebraska, Lincoln, Nebraska, USA), Article-Reports, Daily Quantification of Drought Severity and Duration.

[12] C. Атн. Costantinidis, Bonifica ed Irrigazione: Principi Idrologici, Idraulici ed Agropedologici, Edagricole, (1981).

[13] Taglioli G., Dispense di Irrigazione e Drenaggio, Facoltà di Agraria, Bologna.

[14] Presidenza del Consiglio Dei Ministri, Servizio Idrografico e Mareografico Nazionale, Bollettini $e$ Annali Idrologici.

Parole chiave: Cambiamenti climatici, Conseguenze, Drenaggio, Irrigazione.

\section{SUMMARY}

\section{CLIMATIC CHANGES AND EFFECTS ON IR- RIGATION AND DRAINAGE}

In the last years, many scientists have discussed about climatic changes.

In Italy an important decline of precipitation and a general increase of temperatures were registered at the end of the last century.

The study of precipitations over a 65 years period in a part of the Province of Ferrara confirmed this trend. The SPI index was calculated with the rainfall data. The results of this study point out that we are going toward a period of drought, especially as regard of stratum water resources.

Another important fact is the increase of evapotraspiration in summer periods.

Finally, we demonstrate that an increase in rain intensity will lead to a necessary change in the distance between ditches of about $20 \%$. In the future, careful attention must be addressed to irrigation and drainage in order to ensure good agriculture practices.

Key words: Climatic changes, Effects, Irrigation, Drainage. 